\title{
Towards thermal model of automotive lamps
}

\author{
by M. Dauphin ${ }^{(*, * *, 1)}$, S. Albin ${ }^{(*)}$, M. El Hafi ${ }^{(*)}$, Y. Le Maoult ${ }^{(*)}$, F.M. Schmidt ${ }^{(*)}$
}

${ }^{(*)}$ Université de Toulouse ; Mines Albi, ICA (Institut Clément Ader);

Campus Jarlard, F-81013 Albi cedex 09, France

${ }^{(*)}$ Valeo Lighting Systems ;

34, rue Saint-André, 93000 Bobigny, France

(1) corresponding author : myriam.dauphin@valeo.com ; myriam.dauphin@mines-albi.fr

\section{Abstract}

Automotive lighting systems are constituted of light sources and projection lens system. Thermal effects may induce hot spots on the reflectors and screens. It is essential that temperature of these hot spots doesn't exceed the degradation temperature level of materials. The crucial issue of our work is to model properly the interaction between the radiation emitted by several lamps and the enclosed chamber. In order to develop a reliable numerical model, pertinent input data are required such as filament and glass bulb temperatures. In this study, we focus on the infrared heating modelling of a specific incandescent lamp. As the filament is the main source of heat transfers, an approach is presented for its temperature determination: the "resistivity" method coupled with an optical method.

\section{Introduction}

The design of headlights optics (reflectors, cover glasses) may induce focusing of rays on plastic components of the automotive lighting system. These points of high radiation concentration cause a local increase of the temperature. We have to ensure, using numerical simulations, that temperature of plastics doesn't exceed their degradation temperature level.

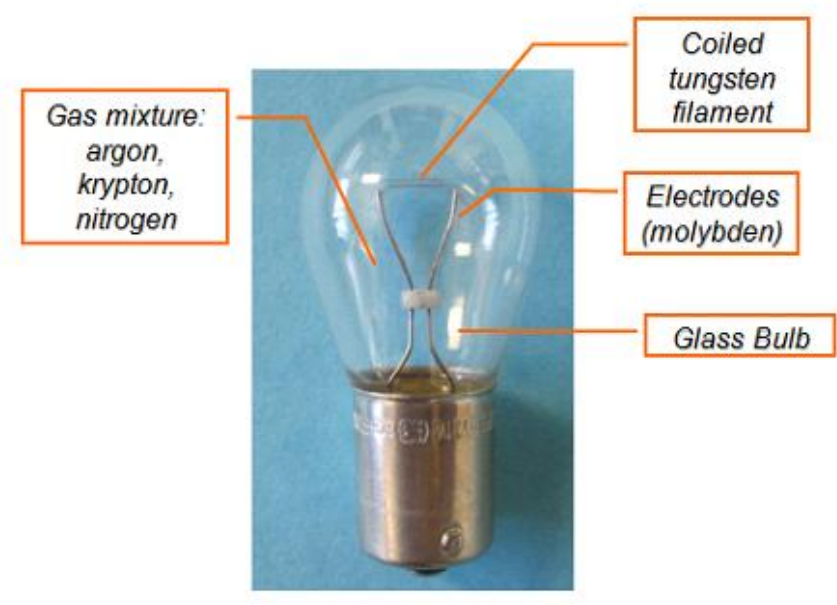

Fig. 1 Incandescent Lamp

The goal of this work is to model accurately the visible and infrared radiation emitted by a specific incandescent lamp P21W type (nominal values: 25W, $13,5 \mathrm{~V})$. The lamp is a complex system constituted of a coiled tungsten filament $(6 \mathrm{~mm}$ length and $0.75 \mathrm{~mm}$ diameter) enclosed in a hard glass bulb filled with a gas mixture (figure.1). Considering the high level temperature of the filament, above $2500 \mathrm{~K}$, it is of prime interest to measure accurately its temperature.

Thus, the literature offers a wide panel of "direct" measurements methods involving high levels of temperatures. These methods can be separated in two categories: methods based on electrical properties of tungsten and methods based on optical measurements. In the first approach, the temperature of the filament is assessed by measuring the electrical resistance of the lamp versus the power delivered by the lamp [1,2]. The methods belonging to the second category are based on techniques using pyrometers. The bi-chromatic pyrometer is commonly used to determine the true temperature of radiative emitters. Instead of considering known values of the spectral emissivity, the ratio of irradiance values collected respectively at two near wavelengths is computed. The source is assumed grey for these wavelengths so that, we are independent from the emissivity [4]. Nevertheless, the measure remains difficult due to strong thermal and spectral dependence of the emissivity coefficient $[5,6]$. Spectrometers enable the collection of radiometric data on a larger spectral bandwidth.

The literature exhibits also analytical approaches where heat transfers balance between different components of the lamp leads to the temperature assessment of both the filament and the bulb $[7,8]$. This heat balance, based on "radiosity approach", requires restrictive conditions. Indeed, we need to assume that the emitter remains at the same time grey, with homogeneous temperature and diffuse emission. 
On the contrary, Monte-Carlo methods applied to radiative transfers take into account a wider variety of physical effects such as spectral and spatial dependence of radiative parameters, specular and diffuse reflections, and light attenuation in semi-transparent media. Indeed, Monte-Carlo method is a powerful method to estimate integrals. So, such physical problem can be formulated by temporal, spatial and spectral integrations. Radiative effects are, with MonteCarlo statistical approach, modelled with density probability functions.

Our software is based on the open source development environment, ED-Star, developed by research team StarWest [9]. In ED-Star, Monte-Carlo method is combined with a rendering tool issued from digital image processing. The rendering tool deals efficiently with complex geometries and thus enables to follow the real light propagation.

In this paper, we have chosen Monte-Carlo method in order to give an appropriate model of both the spatial emission and energetic level of radiation. Thus, in first section, a model is proposed for the tungsten filament. Then, we attempt to highlight the impact of the bulb in the lamp's model. Infrared thermography measurements are used to validate our developments.

\section{The filament: main radiation source}

The radiation power emitted by the filament is given using an integral form (Eq.1). The sampling of the filament's surface gives the emission points. The sampling of the solid angle described by a hemisphere at position $\vec{x}$ gives directions of rays, $\vec{\omega}$.

$\phi_{f}=\int_{S_{f}} \int_{2 \pi} \int_{\lambda=0}^{\lambda=\infty} \varepsilon_{w}\left(\lambda, T_{f}\right) L^{0}\left(\lambda, T_{f}\right) d \lambda(\vec{\omega} \cdot \vec{n}) d \vec{\omega} d \vec{x}$

$S_{f}$ is the surface of the filament, $\varepsilon_{w}$ is the tungsten emissivity, and $L^{0}\left(\lambda, T_{f}\right)$ is the blackbody radiance.

\subsection{Integrated emission assumption}

In a first approach, we opt for an emissivity of tungsten integrated over the wavelength range [0,25 $\mu \mathrm{m} ; 28 \mu \mathrm{m}]$. For example, the integrated emissivity at temperature given by lamp's suppliers, $2900 \mathrm{~K}$, is $\varepsilon_{w}\left(T_{f}\right)=0,30403$. In addition, we assume an isotropic emission and a uniform temperature (Eq.2).

$$
P=R I^{2}=\varepsilon_{w}\left(T_{f}\right) \sigma S_{f}\left(T_{f}^{4}-T_{a m b}^{4}\right) \approx \varepsilon_{w}\left(T_{f}\right) \sigma S_{f} T_{f}^{4}
$$

The ratio between $T_{f}$ and $T_{a m b}$ ensures that the ambient temperature is negligible.

$\left(\frac{T_{a m b}}{T_{f}}\right)^{4}=\left(\frac{433}{2900}\right)^{4}=5 \times 10^{-4}$, where the ambient temperature is here the temperature of the glass bulb: $T_{a m b}=T_{b}$.

In these conditions, the power of the lamp would be sufficient as input energetic parameter of our numerical model (Eq.3).

$$
\phi_{f}=\frac{\varepsilon_{w}\left(T_{f}\right) \sigma T_{f}^{4}}{\pi} \int_{S_{f}} \int_{2 \pi}(\vec{\omega} \cdot \vec{n}) d \vec{\omega} d \vec{x}=\frac{P}{\pi} \int_{2 \pi}(\vec{\omega} \cdot \vec{n}) d \vec{\omega}
$$

\subsection{Spectral emitter}

In a second step, spectral integration will be necessary in order to improve the numerical model. The power of the lamp is no more sufficient; the temperature of the filament is necessary as an input data. The relation between power of the lamp and temperature of the filament is presented in the next section.

\subsubsection{Resistivity method}

Preliminary experiments have consisted in measuring the "resistivity" temperature, $T_{r}$, of the tungsten filament from electrical measurements. A classical law [1,2] links the electrical resistance to the resistivity of tungsten which is an intrinsic property of materials (Eq.4). 
$\left[\frac{R\left(T_{r}\right)}{R_{0}}\right]_{\text {mod el }}=\beta \frac{\rho_{w}\left(T_{r}\right)}{\rho_{w 0}}$

where, $\beta$, assumed to remain constant, indicates the dilatation of the filament such as: $\beta=\frac{\left(L_{f} / S_{f}\right)_{T_{f}}}{\left(L_{f} / S_{f}\right)_{T_{a m b}}}=0.987$.

The data interpolated for pure tungsten [3] gives a polynomial law of resistivity versus temperature (Eq.5).

$$
\rho_{w}\left(T_{r}\right)=1,7348.10^{-6} T_{r}^{2}+2,5665.10^{-2} T_{r}-2,5095
$$

A least square method is used to calculate the filament temperature. According to figure 2, electrical measurements are consistent with the measures issued from literature [1,2]. It is to be noted that the resistivity method is less accurate for low power values. Indeed, the method is very sensitive to the measurement of the resistance at ambient temperature $R_{0}$. Minimizing the resistance of wire connections will improve the measure of $R_{0}$.

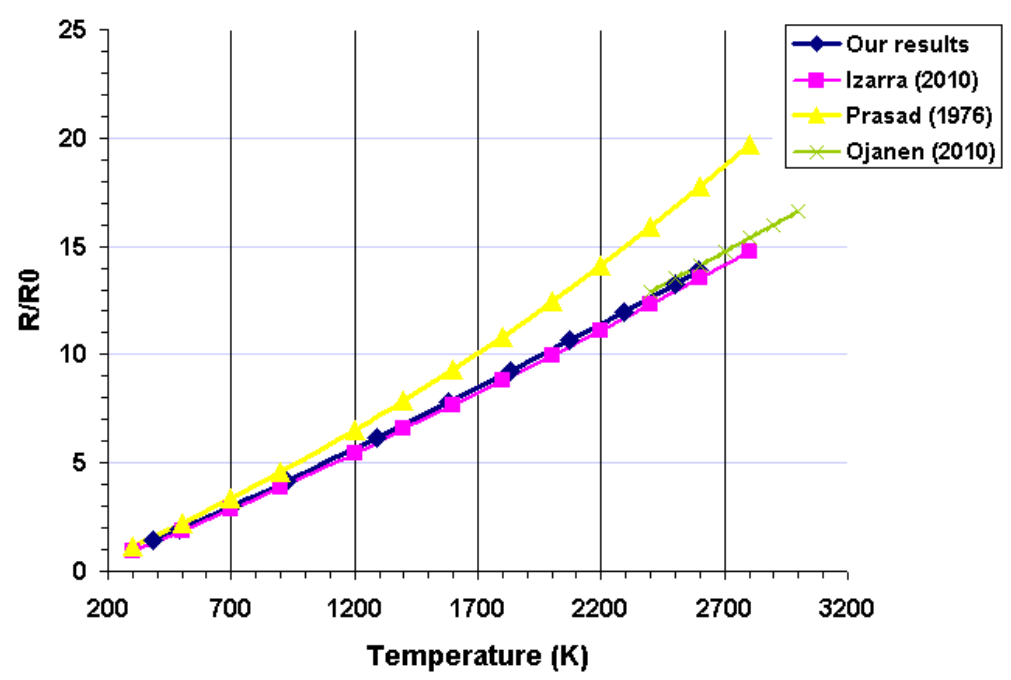

Fig. 2 Electrical resistance versus temperature of filament (P21W lamp)

Ling $\mathrm{Fu}[10]$ has measured the temperature distribution along the filament of a 20W-halogen lamp and found that temperature distribution is inhomogeneous. Thus, we have developed an experimental set-up in order to measure the brightness profile of the tungsten filament (figure 3.a).

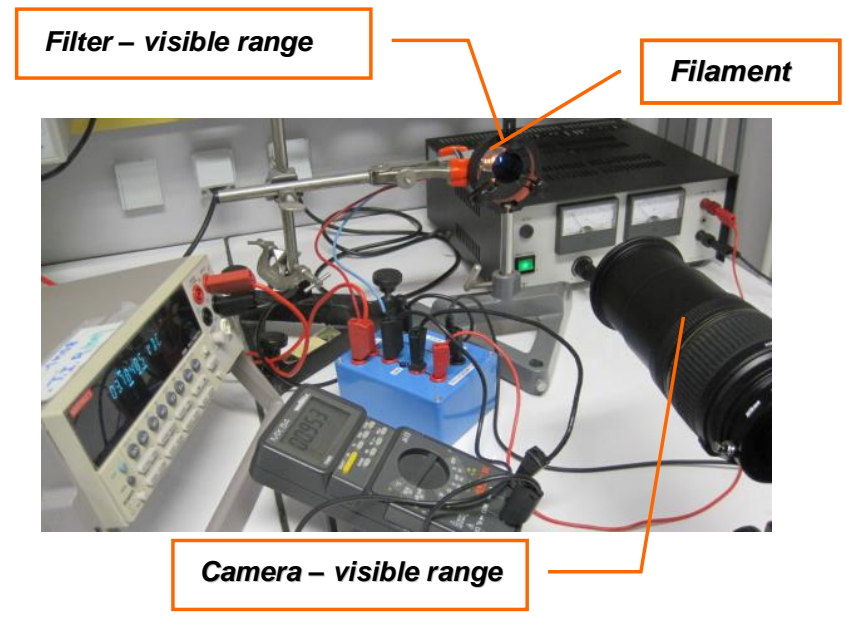

Fig. 3a. Set-up for the filament brightness measurement

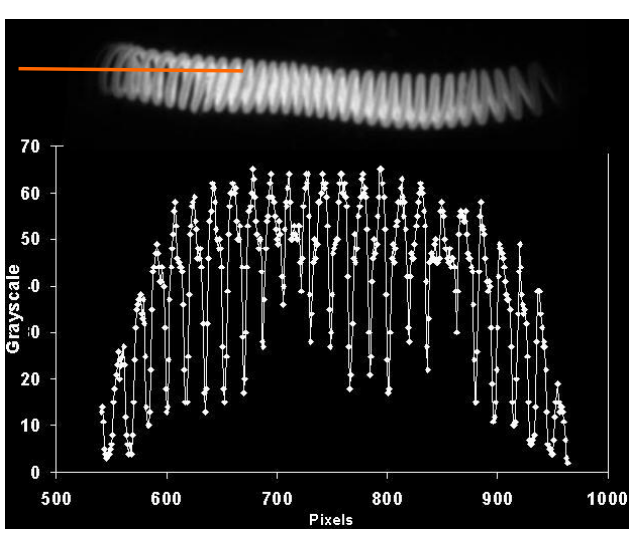

Fig. 3b. Brightness profile 
Figure 4, the emissivity is plotted versus wavelength for different temperature $[5,6]$. It is important to note that emissivity exhibits large variation versus wavelength as well as temperature.

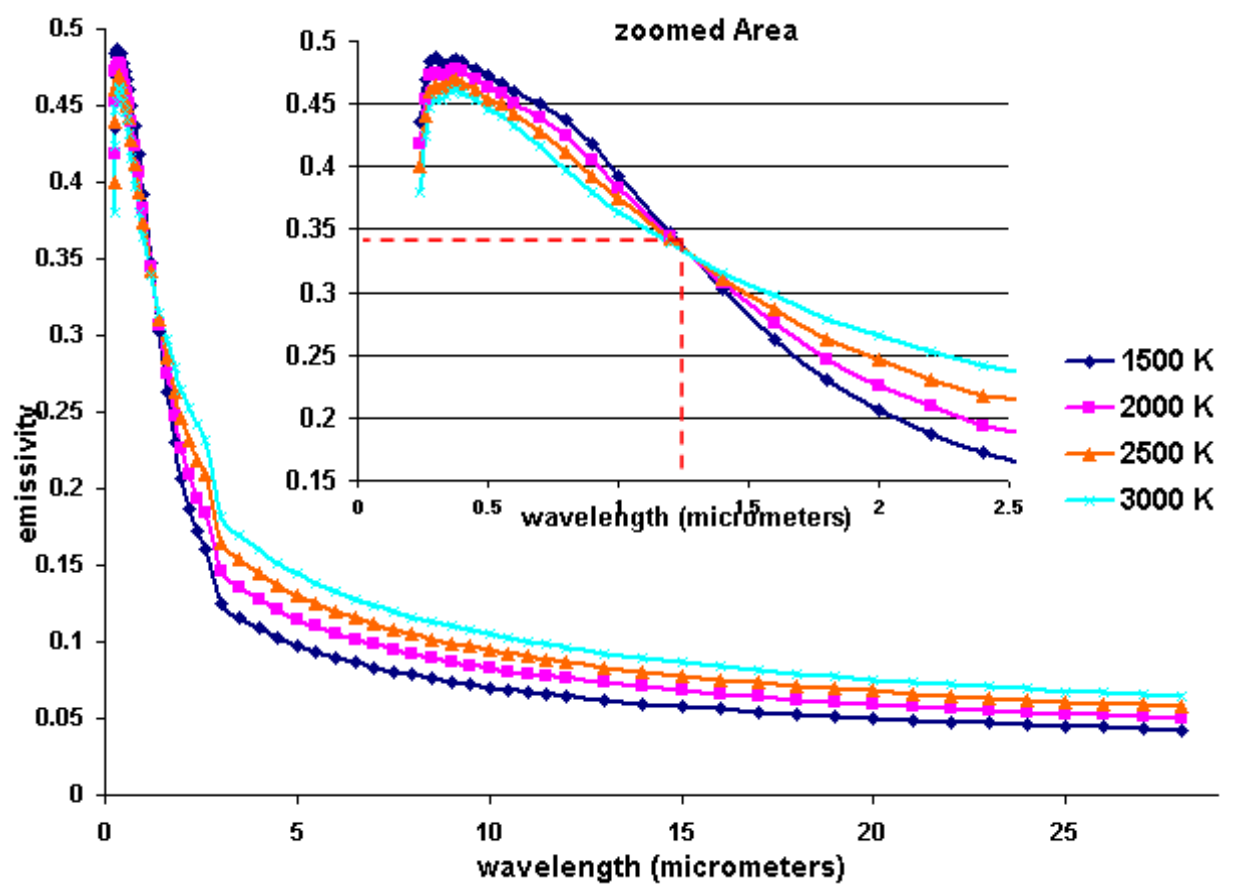

Fig. 4 Tungsten Emissivity data - Desvignes [5], Hagen-Rubens [6]

Consequently, we need to use a narrow-bandwidth filter centered at 0,46 $\mu \mathrm{m}$ (bandwidth at half-heigt of $0,0453 \mu \mathrm{m}$ ) where the emissivity is assumed constant with wavelength. In addition, between $2600 \mathrm{~K}$ and $3000 \mathrm{~K}$, emissivity variation is of the order $1,55 \%$. Using this specific filter, we can assume that the brightness decrease is only caused by a temperature variation of the filament (figure 3.b) and not by a variation of the emissivity. In order to ensure complete independence with respect to the emissivity, we should prefer a narrowband filter centered precisely at the intersection of the curves, close to $1,3 \mu \mathrm{m}$ (figure. 4).

Considering variations observed on brightness profile (figure 3.b), it seems that resistivity temperature represents an average temperature. We will further develop a method permitting to evaluate an "optical" temperature of filament $\mathrm{T}^{\mathrm{opt}}$ necessary in our radiation model.

\subsubsection{Resistivity method coupled with the brightness profile of the filament}

In this section, we attempt to determine the appropriate correction for $T_{r}$. The grey levels $I_{N_{i}}$ are identified using the brightness profile (figure 3.b). The absolute radiance is derived from Planck's law (Eq.6). $K$ is a constant which includes the sensitivity of the detector, the optical constants relating to the camera, and the spectral response, $\chi_{\delta \lambda}$, of the detector. Keeping in mind that the tungsten emissivity is a thermal-dependent parameter, we deliberately assume that using our filter, the emissivity $\varepsilon_{\delta \lambda}$ is constant between the maximum temperature and the minimum temperature of the filament's profile.

$$
I_{N_{i}}=K \varepsilon_{\delta \lambda} c_{1} \lambda_{f}^{-5} \exp \left(\frac{-c_{2}}{\lambda_{f} T^{o p t}(i)}\right)
$$

Using the same equation for the maximum radiance $I_{N_{\max }}$ and then calculating the logarithm of the ratio $\frac{I_{N_{i}}}{I_{N \max }}$, leads to the following equation (Eq.7): 


$$
\ln \left(\frac{I_{N_{i}}}{I_{N \max }}\right)=\frac{c_{2}}{\lambda_{f}}\left[\frac{1}{T^{o p t}{ }_{\max }}-\frac{1}{T^{o p t}(i)}\right]
$$

The brightness profiles are identified for a set of four lamps. The curves are averaged and normalised. The overlaying of curves means that this physical effect is reproducible (figure 5a.).

By setting the maximum temperature, it is possible to deduce the entire profile $T^{\text {opt }}$ (i)(Eq.8) as shown in figure 5b. In a first approach, we chose the temperature of the lamp supplier: 2900K [11].

$$
\frac{1}{T^{\text {opt }}(i)}=\frac{1}{T_{\text {max }}^{\text {opt }}}-\frac{\lambda_{f}}{c_{2}} \ln \left(\frac{I_{N i}}{I_{N \max }}\right)
$$

Assuming that $2900 \mathrm{~K}$ is the maximum temperature, $T_{r}$ needs to be corrected in relation to the average temperature from the profile $\bar{T}^{o p t}(i)$ using a multiplicative factor defined as:

$\bar{T}^{\text {opt }}(i)=\alpha T_{r}$ where $\alpha=1,09679$

Otherwise, assuming that the average temperature of the brightness profile is equal to the temperature "resistivity", we obtain only a maximum value for the filament up to $2644 \mathrm{~K}$.

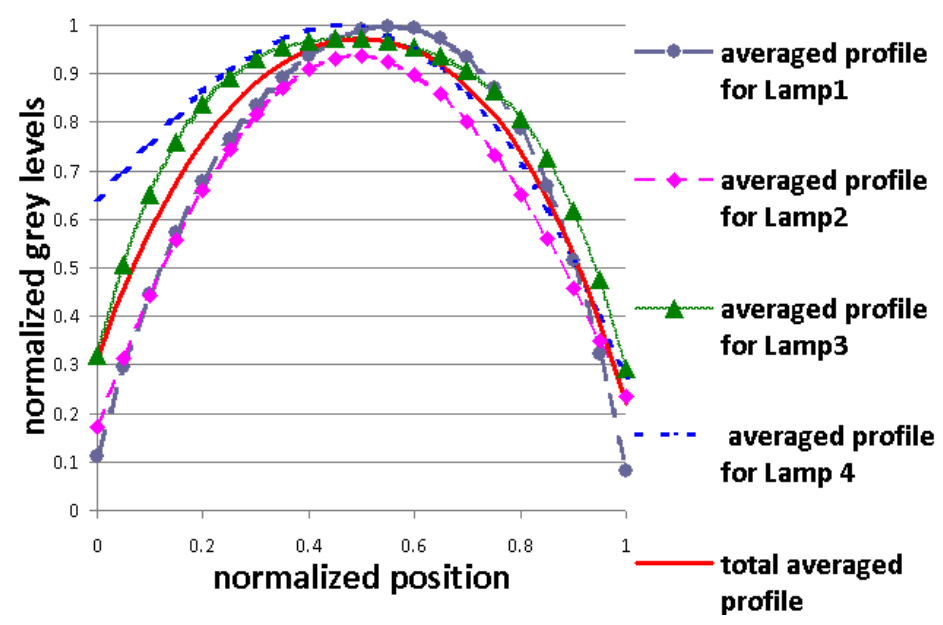

Fig. 5a. Average profiles of brightness

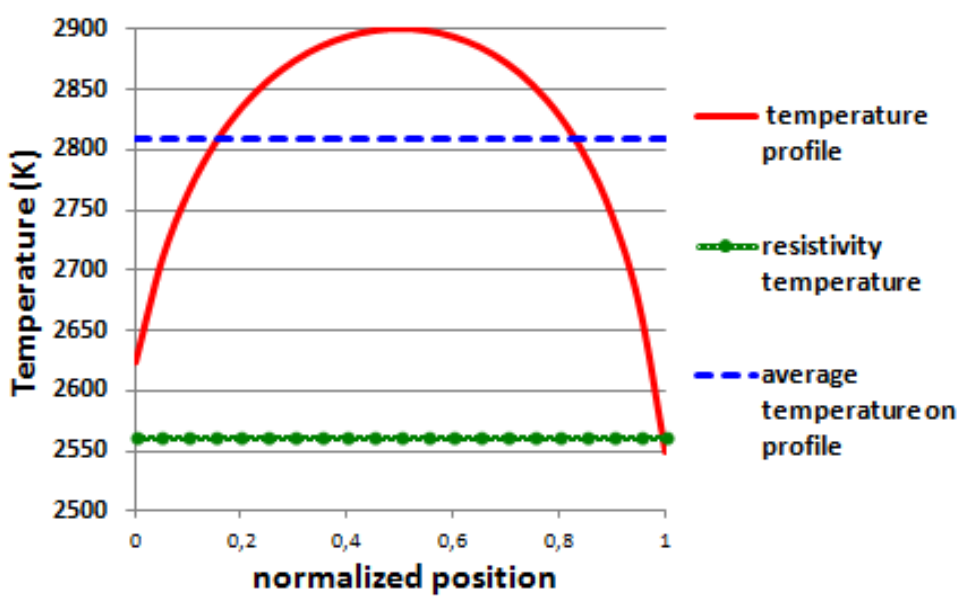

Fig. 5b. Temperature profile

Such a difference between "resistivity" temperature and average temperature from profile can be due to the blue filter employment which doesn't enable to detect the lowest intensity levels on filament ends, leading thus to the 
overestimation of the mean value of the profile. Ideally, we should prefer a filter centred at $1.3 \mu \mathrm{m}$, where the camera gets a higher signal/noise ratio and consequently access the minimum level.

\subsection{Geometrical model : point source assumption}

An analytical study gave us a comparison of normal irradiance $q_{N p}$ obtained on a surface plate irradiated by a point source and by an extended source $q_{N d}$ (disk of radius R)(Eqs.10 and 11).

$q_{N p}=\frac{l}{D^{2}}$

where $D$ is the distance between the source and the plate and I is the radiant intensity.

$q_{N d}=\frac{l}{D^{2}}\left[\frac{1}{1+\left(\frac{R}{D}\right)^{2}}\right]$

Plotting $\left|\frac{q_{N d}}{q_{N p}}-1\right|$ versus R/D ratio, demonstrates that the source point assumption for the filament is only valid for $D \geq 10 R$. That is to say $30 \mathrm{~mm}$ for a filament of $6 \mathrm{~mm}$ length or for a disk of $6 \mathrm{~mm}$ diameter. In conclusion, the point source model can be used for the filament assuming a distance greater than $30 \mathrm{~mm}$. Thus, for the numerical simulations performed in section 3.2, we will use $D=65 \mathrm{~mm}$, avoiding also the deterioration of the test sheet.

\section{Numerical model of the lamp}

In this part, we will implement the complete model of the lamp including both the tungsten filament and glass bulb. The importance of the glass bulb in this model can be assessed by comparing numerical computation and temperature measurements using an infrared camera obtained on an ABS sheet irradiated by the lamp.

\subsection{Glass bulb temperature measurement using infrared camera}

According to the apparent transmission curve of the glass bulb (IRTF Bruker Vertex70 spectrometer), the bulb absorbs a small fraction of the blackbody emission given by Planck's law at the filament's temperature (figure 6.b). Thus, the glass bulb has to be considered in the model as a secondary emitting source because of a high ratio between surfaces of the glass bulb and the filament, $\frac{S_{b}}{S_{f}} \approx 100$.

Infrared camera is used to measure its surface temperature distribution. The detector is sensitive in the spectral band [8 $12 \mu \mathrm{m}]$, where the glass bulb is opaque with respect to the radiation emitted by the filament. Thermogram (figure 6.a) highlights its non-homogeneity due to convective phenomena inside the glass bulb due to the presence of gas, and around the glass bulb in the enclosed space.

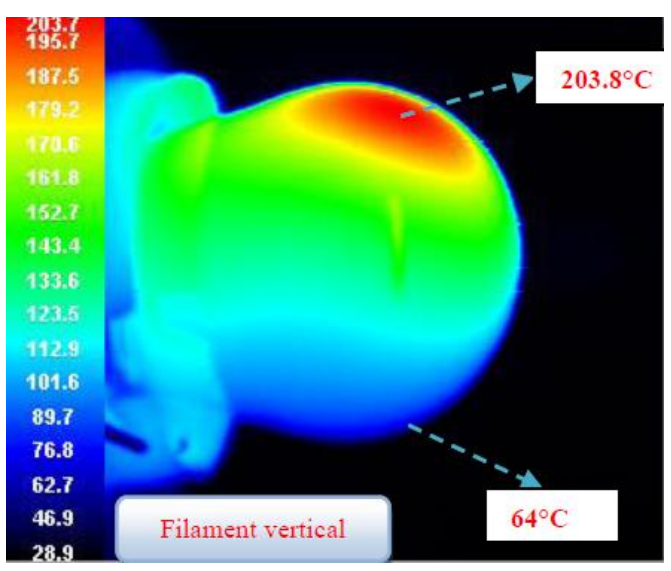

Fig. 6a. Infrared camera image of the bulb $(P=23,8 W)$

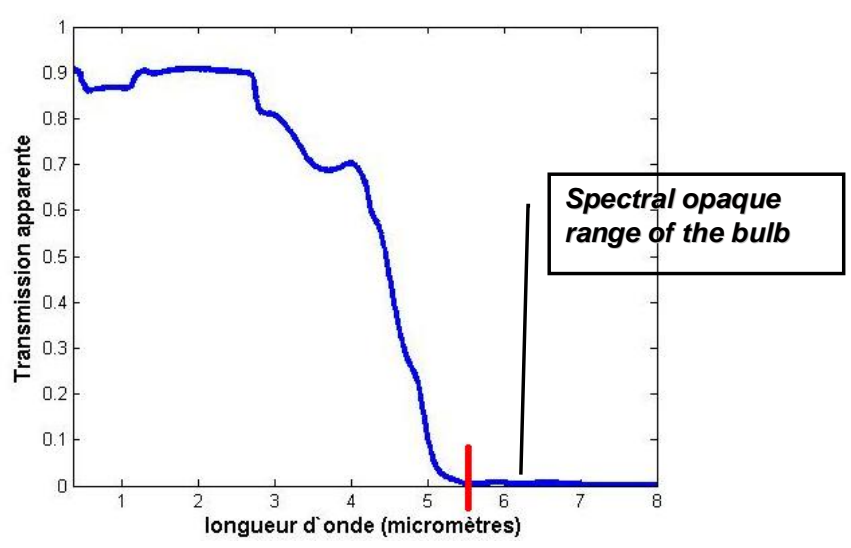

Fig. 6b. Bulb's transmission curve 
In this section, we have listed different radiative characteristics of the source. In order to develop the numerical model, we have referred main parameters for both ray-tracing and thermal simulations (Table 1, Table 2).

Table 1. Filament's parameters for ray-tracing computation

\begin{tabular}{|c|c|}
\hline Parameters & Values \\
\hline Length $\mathrm{L}_{\mathrm{f}}$ & $6(\mathrm{~mm})$ \\
\hline Radiation & Isotropic \\
\hline $\mathrm{P}_{\mathrm{f}}=\mathrm{R}^{2}$ & $23,8 \mathrm{~W}$ \\
\hline
\end{tabular}

Table 2. Glass bulb's parameters for ray-tracing computation

\begin{tabular}{|c|c|}
\hline Parameters & Values \\
\hline Geometry & Spherical \\
\hline Diameter $\mathrm{d}_{\mathrm{b}}$ & $25 \mathrm{~mm}$ \\
\hline Radiation & Isotropic \\
\hline Average temperature $\mathrm{T}_{\mathrm{b}}$ at $\mathrm{P}_{\mathrm{f}}$ & $433 \mathrm{~K}$ \\
\hline Emissivity $\varepsilon_{\mathrm{b}}$ & 0,85 \\
\hline
\end{tabular}

Measurements using infrared thermography on the irradiated sheet may be used as a mean to perform direct validation and adjust input data of our numerical model. A fine three-coefficients calibration of our IR-Camera (Thermovision 880 , 8-12 $\mu \mathrm{m}, 280$ lines $\times 280$ points) has been performed:

The calibration has been done from $30^{\circ} \mathrm{C}$ until $160^{\circ} \mathrm{C}$, by step of $5^{\circ} \mathrm{C}$ on the range $30-60^{\circ} \mathrm{C}$, and by step of $10^{\circ} \mathrm{C}$ above . The small remaining difference between the blackbody temperature (LANDCAL- $550^{\circ} \mathrm{C}$ ) and its temperature measured by thermography within the range $30-60^{\circ} \mathrm{C}$ is $\Delta T \approx 0,1^{\circ} \mathrm{C}$. This confirms the good accuracy of the calibration. In order to limit any bias induced by thermography, we chose, for all studies recounted throughout this paper, a testing sample whose characteristics are well known and referenced in the table 3.

Table 3. parameters for an ABS-N plate

\begin{tabular}{|c|c|}
\hline Parameters & Values \\
\hline Thickness & $1,4(\mathrm{~mm})$ \\
\hline Density $\rho_{\mathrm{m}}$ & $1050\left(\mathrm{~kg} / \mathrm{m}^{3}\right)$ \\
\hline Thermal conductivity $\mathrm{K}$ & $0,10(\mathrm{~W} / \mathrm{m} \cdot \mathrm{K})$ \\
\hline Specific heat $\mathrm{c}_{\mathrm{p}}$ & $1680(\mathrm{~J} / \mathrm{kg} \cdot \mathrm{K})$ \\
\hline Emissivity $\varepsilon_{\text {ABS }}$ & 0,9 \\
\hline
\end{tabular}

\subsection{Radiation computation}

Due to the glass bulb, the assumption of source point is not valid for the lamp. In addition, the surface temperature distribution of the glass bulb is not uniform. Thus, the ray-tracing tool is of great interest to model accurately the spatial distribution of radiance emitted by the glass bulb [12].

Computations have been performed in order to compute the flux density on an opaque ABS sheet irradiated by a P21W lamp [13]. The experimental set-up is described figure 7. 


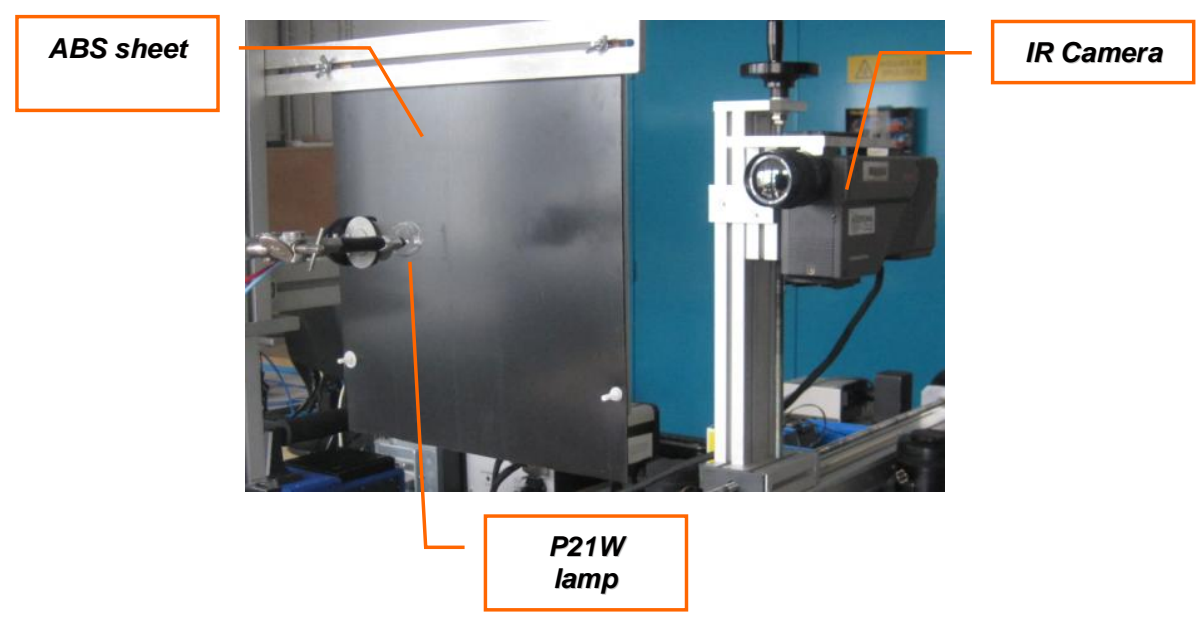

Fig. 7. Experimental set-up for opaque sheet irradiation

In a first approach, the glass bulb emissivity is integrated on the wavelength range [ $8 \mu \mathrm{m}, 12 \mu \mathrm{m}]$. We assume also an average temperature $T_{b}=433 \mathrm{~K}$, in accordance to the thermal distribution of the bulb (figure $6 \mathrm{a}$ ).

The radiation contribution of the glass bulb, received on the ABS sheet, is added to the contribution of the filament so that flux density profiles are compared: $\vec{q}_{\text {total }}(r)=\vec{q}_{f}(r)+\vec{q}_{b}(r)$. The emission of the filament and the bulb are handled separately. Thus, the effects of light attenuation through the bulb, reflections and refractions on dioptre are not yet modelled. So, it is possible to evaluate the effect of the bulb in relative proportions (figure 8). The figure 8 shows a contribution of the bulb on the $\overrightarrow{\mathrm{q}}_{\text {total }}(r)$, up to $14 \%$ on the maximum value.

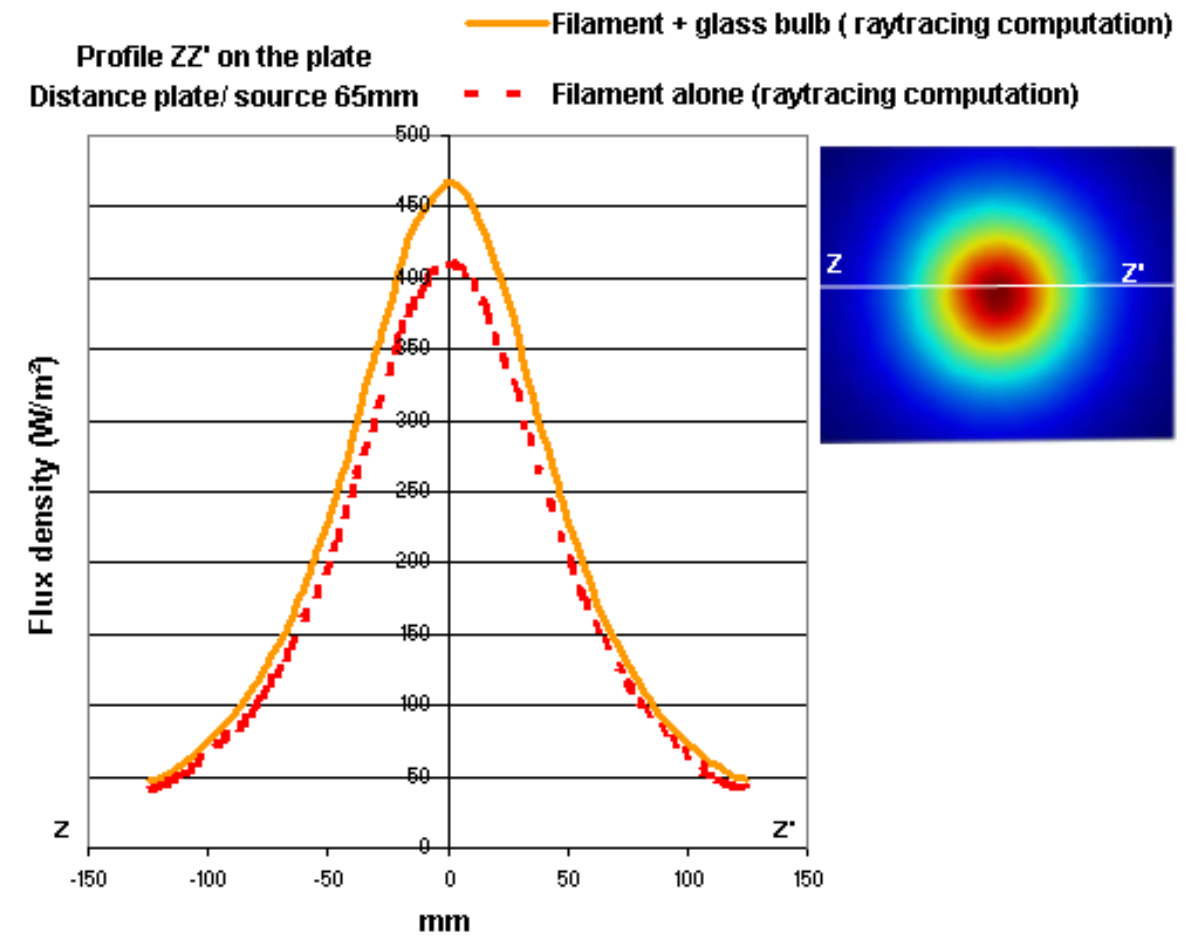

Fig. 8. Effect of the glass bulb on density flux distribution

\subsection{Thermal simulation}

The flux density distribution obtained using ray-tracing tool is considered as a boundary condition on front face of the thermoplastic sheet. 3D thermal computations performed using Comsol Multiphysics software are compared to 
experimental temperature measured on the back surface of the sheet. Temperature profiles (filament with and without glass bulb) have been compared (figure 9).

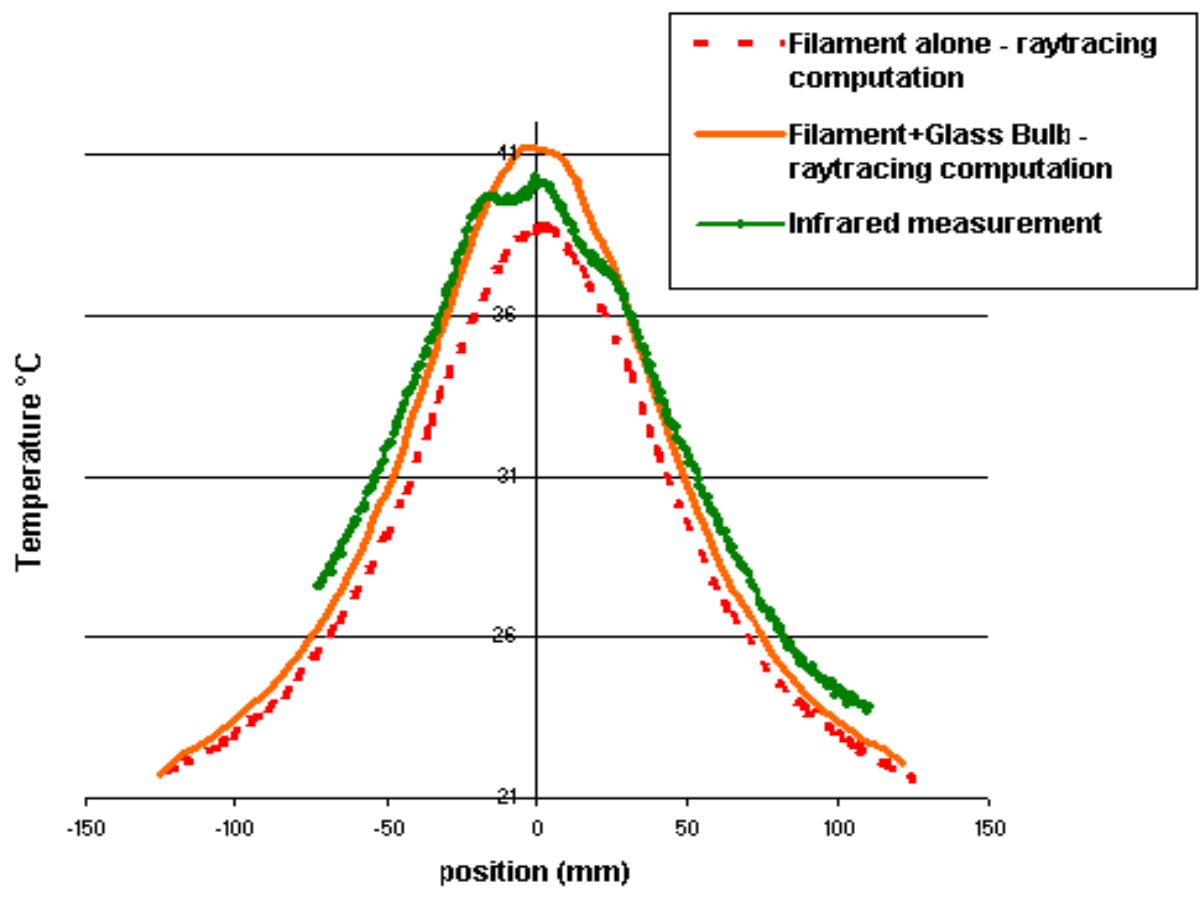

Fig. 9. Comparison between measured and computed temperature profiles

Numerical model using filament alone underestimates the temperature profile $(\sim 2.5 \%$ error for maximum temperature). In addition, the model including the glass bulb overestimates the temperature in the same proportion. So, the real maximal temperature is bounded by the two computations. The curve obtained with the model including the filament and the glass bulb fits better the experimental data between positions $30 \mathrm{~mm}$ and $50 \mathrm{~mm}$. Thus, we are able to conclude that the spatial emission of the lamp is better modelled by taking into account the specific emission of the bulb.

We notice a defect on the maximum value of the thermogram caused by refraction effects of the glass bulb. This observation suggests that the polar emission of the filament through the glass bulb can not be faithfully reproduced using a point source.

\section{Conclusions and future work}

In the light of the results presented in this article, the filament can be modelled by a point source whose temperature is equal to a corrected "resistivity" temperature measured at a given power. Further studies will be conducted to test limit cases of distances between the filament and the receiver. Furthermore, the impact of the bulb on total heat balance has been demonstrated.

We will improve our extended "resistivity" method using the adequate filter centred at the intersection of the tungsten emissivity curves, as mentioned in section 2.2. In order to improve data of the filament's average temperature, a specific experimental set-up is being designed to measure the absolute radiance emitted from the lamp. The overall set-up includes a USB-Spectrometer and a calibrated tungsten-halogen light source, whose colour temperature, 3100K, is perfectly known.

As we have performed direct comparison, we will develop an "inverse method". The "inverse method" consists in fitting simulated temperature distribution and values measured by thermography on the ABS opaque sheet by an optimisation loop. The filament temperature is an input parameter which is modified automatically to reach the objective, i.e. minimize the variation between simulations and experimental data.

Finally, efforts will focus on the improvement of the bulb's model. The Integration of the real geometry (CAD) and the temperature variation of the bulb will allow a better consideration of the bulb's radiation contribution as a secondary emitting source. A better agreement with thermographic measurements is expected. Finally, our raytracing tool will be extended to take into account radiative properties of the automotive lighting system, such as reflector's specular 
reflections and the optical path of rays crossing through semi-transparent media, among them, the bulb and glasses. The thermography is always used to validate the models developed.

\section{REFERENCES}

[1] Izarra C., Gitton J.M., "Calibration and temperature profile of a tungsten filament lamp", European Journal of Physics, 2010

[2] Ojanen M., Karha P., Ikonen E., "Spectral irradiance model for tungsten halogen lamps in 340-850 nm wavelength range", Applied Optics , 2010

[3] Smithells J., "Smithells metals reference book". W.F. Gale \& T.C. Totemeir, 2004

[4] Lechner W., Schob, O. " Temperature measurement of filaments above $2500 \mathrm{~K}$ applying two-wavelength pyrometry ", Institute of Physics Conference Series, 26 :297-305, 1975

[5] Devignes F., Propriétés des rayonnements optiques. Masson, 1997 (in French)

[6] Siegel R., Howell J.R., "Thermal Radiation Heat Transfer", Hemisphere publishing corporation, 3 edition, 1992

[7] Pettersson M., Stenström S. "Modelling of an electric IR heater at transient and steady state conditions", International Journal of Heat and Mass Transfer, $43: 1209-1222,2000$

[8] Virag M., Murin J. "Thermal field simulation of a tungsten filament lamp referring to its lifetime", Journal of electrical engineering, 56(9-10):252-257, 2005

[9] Groupe de recherche Star-West. Ed-Star (environnement de développement pour les statistiques radiatives). http://wiki-energetique.laplace.univ-tlse.fr/wiki/index.php/starwest. (in French)

[10] Fu L., Leutz R., Ries H. "Physical modeling of filament light sources", Journal of Applied Physics ",100 [103528.1103528.6], 2006

[11] Philips internal report, "Thermische Analyse P21W"

[12] Cosson B., "Infrared heating stage simulation of semi-transparent media (PET) using ray tracing method", International Journal of Material Forming, 2010

[13] Halelfadl S., "Modélisation des transferts thermiques dans un feu de signalisation de voiture", In french, Master Degree, Ecole des Mines d'Albi Carmaux, 2010

\section{ACKNOWLEDGMENTS}

The authors would like to acknowledge Valeo Lighting Systems for having supported this work. 